\title{
Perspectives Of Health Care Professionals On Patient Involvement In Patient Safety At Tertiary Care Women's Hospitals In Colombo District
}

\author{
Dr W D K Wijesinghe *, Dr Sathasivam Sridharan ** \\ * Trainee, MD in Medical Administration, Post Graduate Institute of Medicine. University of Colombo. \\ ** Deputy Director General - Planning, Ministry of Health, Colombo, Sri Lanka \\ DOI: 10.29322/IJSRP.11.09.2021.p11716 \\ http://dx.doi.org/10.29322/IJSRP.11.09.2021.p11716
}

\begin{abstract}
Introduction : In modern medicine, there's an important recognition on patient safety culture and patient's participation in treatment procedure. However, very little known about health care professionals' attitude on patient participation in such patient safety related procedures and also hardly any research is done on our country up to now.
\end{abstract}

Methodology: This is a hospital-based descriptive, crosssectional study were carried out in tertiary care Women's hospitals in the Colombo district. The study were carried out from $10^{\text {th }}$ November 2016 to $22^{\text {nd }}$ August 2017.

Three hundred and ninety-one health-care professionals were participated in this study. (94 doctors , 215 nurses, 82 midwives). Stratified random sampling method were used to get the required sample size. The data was collected using a selfadministered questionnaire. . The software package was used for this study was MS-Excel, MS-Access and SPSS 20.

Observations and results: HCPs' attitudes can be varied with type of behavior (interactional and noninteractional behavior), type of HCP category and participants own professional role.In general all three selected HCPs' were had positive attitudes towards patient safety . However, incident reporting component showed negative attitude among all three selected HCPs' categories. Components which showed positive attitudes by all HCPs were factual questions, challenging questions ,notifying questions and information provision questions. Over all doctors showed more positive attitudes compare to other two HCP groups in above three components.

Conclusion: our research finding suggest that HCPs' are willing to involve in patient safety behaviours and also they are ready to support patients as a HCP when patients involve in safety related issues. However, further in-depth studies necessary to identify the behavior of different clinical specialties as we were confined to obstetrics and gynaecology.

Index Terms- Patient involvement HCP attitude, Patient safety

\section{INTRODUCTION}

$\mathrm{M}$ odern medicine is a highly complex setup, where service is provided to the clients in a pressurized, constantly evolving technologies with many clinical judgements from health care professionals. There is a very high possibility of clinical assessments can go wrong leading to health hazards to patients. Health hazards can vary from a minor injury to a disaster like death. [1] Error and mistakes in health care is a relatively an old concept. In fact, Hippocrates recognized that harm was possible even after careful consideration about the patient from a skillful healer. Greek healers in the 4th century BC drafted the Hippocratic Oath and pledged to "prescribe regimens for the good of my patients according to my ability and my judgment and never do harm to anyone".[2] An adverse event can be described as an unintended damage to a patient caused by during healthcare provision rather than by the illness itself and which can lead to prolonged hospital stay or to casual or permanent damage to the patient at the time of discharge or both. Adverse events could be due to acts of omission (for an example a failure to identify the disease or provide the correct treatment) and commission (providing wrong treatment).[3]

The Harvard study identified that around 5\% of patients suffered some kind of medical errors during their care. However, majority of harm were temporary injuries but, around $15 \%$ adverse events lead to death. [4]

Nearly 43 million adverse events happen annually around the world due to patient safety related events and cause a staggering 23 million associated disability-adjusted life years. Alarmingly around $66 \%$ of these occur in under develop countries.[5]

Patient safety is one of the core principles of health care. Patient safety is the avoidance of health related mistakes and unwanted events to patients relevant to their care.

Involvement in health care leads to better client satisfaction. Advantages also include higher confidence, lower anxiety, better knowledge requirements, good understanding with heath care professionals. However, patients should not take their care totally into their hands by involving in safety. Patients should work as a bu ffer in addition to those existing safety measurements in a given institution. The current concept of patient management is focused on "shared decision-making" where patients and health care providers are equally involved in decision making process.

There is no clear evidence to say that HCP could support patients when confronting with them in terms of patient safety issues. At the same time, very little or no data are currently available on this topic in Sri Lanka. Therefore, this study is to describe HCP's behavior toward patient safety, as a health care professional . 
Selected Health Care Professionals (HCP) in this study include Consultants, Postgraduate trainees, Senior Medical Officers, Relief House Officers, Intern Medical Officers, Nursing Sisters, Grade Nursing Officers and Midwives.

\section{AIM AND OBJECTIVES}

Aim

To describe the perspectives of selected health care professionals on patient involvement in patient safety at tertiary care women's hospitals in Colombo district as a health care professional

\section{Objectives}

- To describe the socio demographic factors of selected HCP in related to patient involvement in patient safety.

- To determine selected HCP attitudes toward patient involvement in patient safey

- Health Care Professionals perspectives were assessed in 05 different areas.They were as follows. a.To study the selected HCPs attitudes towards factual questions b.To study the selected HCP's attitudes towards challenging questions c. To study the selected HCP's attitudes towards notifying questions $d$. To study the selected HCP's attitudes towards information provision questions e.To study the selected HCP's attitudes towards reporting incidents

\section{RESEARCH METHODOLOGY}

Study of currently available national and international literature on the subject.

Study design: This is a hospital-based descriptive, crosssectional study were carried out in tertiary care Women's hospitals in the Colombo district. There are three tertiary care women's hospitals in Sri Lanka. Of which two are in Colombo district namely De Soysa Hospital for Women (DSHW) and Castle Street Hospital for Women (CSHW). These two hospitals in Colombo district are selected as the study setting.

Participants: Three hundred and ninety-one health-care professionals (94 doctors, 215 nurses, 82 midwives) from Castle Street Hospital for Women and De Soysa Hospital for Women.

- Study area: There are three tertiary care women's hospitals in Sri Lanka. Of which two are in Colombo district namely De Soysa Hospital for Women (DSHW) and Castle Street Hospital for Women (CSHW). These two hospitals in Colombo district are selected as the study setting

- Study tool:

The data was collected using a self-administered questionnaire. Focus group discussion was held on 25th January
2017 at Quality unit at CSHW. Aim of the focus group discussion was to adapt of the questionnaire to suit local conditions. some variables were omitted from the questionnaire used in that study due to inapplicability in the Sri Lankan context. A few variables were added in relation to the working condition and to suit the Sri Lankan context. Attendance sheet of focus group discussion were annexed. Questionnaire -01 was developed to assess the basic socio-demographic characteristics of the participants. Questionnaire- 02 was developed comprising 22 items to assess selected HCP attitudes to support patient involvement in different safety related behaviors. Three main categories of "interactional behaviors" were examined that involve engaging in dialogue with selected HCP: asking factual questions (05 items), challenging questions ( 5 items) and notifying selected HCP problems or errors in their care (04 items). Two categories were used to assess attitude to support "non-interactional behaviors" this include information provision questions (05 items) and incident reporting questions (03 items).

- Study Period:

The study was carried out from 10th November 2016 to 22nd August 2017. Data collection were carried out from 01st of April 2017 to 10th of May 2017 in De Soysa Hospital for Women and Castle Street Hospital for Women. • Unit under Study: The study unit were the main three professional categories who was attached to nine obstetrics and gynaecological units at DSHW and CSHW.

\section{Measures}

Questionnaire -01 was developed to assess the basic socio demographic characteristics of the participants. Questionnaire- 02 was developed comprising 22 items to assess selected HCP attitudes to support patient involvement in different safety related behaviors. Three main categories of "interactional behaviors" were examined that involve engaging in dialogue with selected HCP: asking factual questions (05 items), challenging questions (5 items) and notifying selected HCP problems or errors in their care (04 items). Two categories were used to assess attitude to support "non-interactional behaviors" this include information provision questions (05 items) and incident reporting questions (03 items).

\section{Self-administered questionnaire}

Pre-tested self-administered Questionnaire was used for data collection. Questionnaire will include four sections. Questionnaire 01 - Socio-demographic characteristics. The information collected was on age, gender, professional category and years of experience Questionnaire 02 - Attitudes toward supporting patient involvement (as a selected HCP) Stratified random sampling was carried out based on the duty roster, and respondents were selected. A date for the study was be fixed with the help of the, Consultants, Matron and the Nursing Sister in each hospital. Request were made to the Consultants, Matron and the Nursing Sister of the relevant wards to make necessary arrangements for the selected respondents to be present on the day of survey.

\section{Data Process and Analysis}

The principal investigator was checked the data collected before it was fed into the computer for analysis. The data was 
checked for missing and unused values. The data entry was carried out in SPSS 20 statistical package. Before analysis the computer base was screened for possible data entry errors. Analysis of the data was carried out manually as well as with the aid of computer. The software package was used for this study was MS- Excel, MS-Access and SPSS 20.
Anova test was used to compare selected three HCP groups and post hoc analysis was done using Fisher's Least Significant Difference (LSD) test comparison of two hospitals were done by using independent sample T-test.Scale reliable analysis was done to make sure internal consistency among different groups (Cronbach's $\alpha$ coefficient $>0.7$ ).

IV. RESULTS

Table 1 Participant Characteristics - CSHW and DMHW Hospitals

\begin{tabular}{|c|c|c|c|c|}
\hline Sociodemographic & Doctors & Nurses & Midwives & Total \\
\hline Variables & $n=94(24.04 \%)$ & $\mathrm{n}=215(54.98 \%)$ & $n=82(20.97 \%)$ & $n=391(100 \%)$ \\
\hline Sex Male & 75 (79.8\%) & 0 & 0 & \\
\hline Female & $19(20.2 \%)$ & $215(100 \%)$ & $82(100 \%)$ & \\
\hline \multicolumn{5}{|l|}{ Age } \\
\hline $1(<30$ yrs ) & $46(48.9 \%)$ & $48(22.3 \%)$ & 07 (8.5\%) & $101(25.8 \%)$ \\
\hline 11 (31-40 yrs ) & $25(26.6 \%)$ & $92(42.8 \%)$ & $36(43.9 \%)$ & $153(39.1 \%)$ \\
\hline 111 (41-50 yrs) & $18(19.1 \%)$ & $59(27.4 \%)$ & $29(35.4 \%)$ & $106(27.1 \%)$ \\
\hline $1 \mathrm{~V}(>51 \mathrm{yrs})$ & 05 (5.3\%) & $16(7.4 \%)$ & $10(12.2 \%)$ & $31(7.9 \%)$ \\
\hline \multicolumn{5}{|l|}{ Professional category } \\
\hline Consultants & & 05 (1.3\%) & & \\
\hline Post Graduate Trainee & & $10(2.6 \%)$ & & \\
\hline Senior House Officer & & $35(9 \%)$ & & \\
\hline Resident House Officer & & $18(4.6 \%)$ & & \\
\hline Intern House Officer & & $26(6.6 \%)$ & & \\
\hline Ward Sister & & $07(1.8 \%)$ & & \\
\hline Grade Nursing Officer & & $208(53.2 \%)$ & & \\
\hline Midwife & & $82(21 \%)$ & & \\
\hline \multicolumn{5}{|c|}{ Total Working experience } \\
\hline \multicolumn{5}{|c|}{ Years } \\
\hline$<5$ & $48(51.1 \%)$ & $34(15.8 \%)$ & $18(22 \%)$ & $100(25.6 \%)$ \\
\hline $6-10$ & $17(18.1 \%)$ & $73(34.0 \%)$ & $20(24.4 \%)$ & $110(28.1 \%)$ \\
\hline $11-15$ & $20(21.3 \%)$ & $47(21.9 \%)$ & 19 (23.2\% & $86(22 \%)$ \\
\hline $16-20$ & 04 (4.3\%) & $46(21.4 \%)$ & $16(19.5 \%)$ & 66 (16.9\%) \\
\hline $21-25$ & $02(2.1 \%)$ & 07 (3.3\%) & $08(9.8 \%)$ & $17(4.3 \%)$ \\
\hline$>26$ & 03 (3.2\%) & 08 (3.7\%) & 01 (1.2\%) & $12(3.1 \%)$ \\
\hline
\end{tabular}

Working experience in current institution

Years

$\begin{array}{lllll}<5 & 92(97.9 \%) & 127(59.1 \%) & 42(51.2 \%) & 261(66.8 \%) \\ 6-10 & 00(0.0 \%) & 74(34.4 \%) & 24(29.3 \%) & 98(25.1 \%) \\ 11-15 & 01(1.1 \%) & 10(4.7 \%) & 13(15.9 \%) & 24(6.1 \%) \\ 16-20 & 01(1.1 \%) & 04(1.9 \%) & 03(3.7 \%) & 08(2.0 \%) \\ \begin{array}{l}\text { Qualifications } \\ \text { Diploma }\end{array} & 00(0.0 \%) & 00(0.0 \%) & 82(100 \%) & 82(21 \%) \\ \text { Basic Degree } & 00(0.0 \%) & 215(100 \%) & 00(0.0 \%) & 215(55 \%)\end{array}$


Undergraduate Degree

$89(94.7 \%)$

$05(5.3 \%)$
$00(0.0 \%)$

$00(0.0 \%)$
$00(0.0 \%)$

$00(0.0 \%)$
$89(22.8 \%)$

$05(1.3 \%)$
Table 1 shows participant characteristics. Altogether there were 391 respondents. (Total sample was 422 with a $92 \%$ response rate). There were $94(24 \%)$ doctors, $215(55 \%)$ nurses and $82(21 \%)$ midwives for the study as a whole. Age distribution showed that around $50 \%$ of doctors were in less than 30 years' age category. major proportion of nurses and midwives were in age 31- 40 category, $43 \%$ and $44 \%$ respectively. Majority of doctors $48(51 \%)$ had total working experience, in contrast nurses and midwives more spread in total working experience. Current working experience showed that majority of three selected HCPs' were in less than 05 years' category. Professional qualification showed that all midwives holding a diploma (100\%), nurses were holding a basic degree $(100 \%)$ and majority of doctors holding an undergraduate degree $(95 \%)$.

\section{Descriptive Statistics for Survey : Attitudes Toward Supporting Patient Involvement as a HCP}

Table 2 - Descriptive statistics for selected HCPs attitudes towards factual questions as a $\mathrm{HCP}$ in CSHW and DSHW hospitals

\begin{tabular}{|c|c|c|c|c|c|c|}
\hline \multirow[t]{3}{*}{ Item Description } & Doctors & Nurses & Midwives & Total & $\mathbf{F}$ & $P$ value \\
\hline & $\begin{array}{l}n=94 \\
(24 \%)\end{array}$ & $\begin{array}{l}n=215 \\
(55 \%)\end{array}$ & $\begin{array}{c}n=82 \\
(21 \%)\end{array}$ & $\begin{array}{l}N=391 \\
(100 \%)\end{array}$ & & \\
\hline & $\begin{array}{l}\text { Mean } \\
\text { SD }\end{array}$ & $\begin{array}{l}\text { Mean } \\
\text { SD }\end{array}$ & $\begin{array}{l}\text { Mean } \\
\text { SD }\end{array}$ & $\begin{array}{l}\text { Mean } \\
\text { SD }\end{array}$ & & \\
\hline \multicolumn{7}{|l|}{$\begin{array}{l}\text { Factual questions (interactional } \\
\text { behavior) }\end{array}$} \\
\hline $\begin{array}{l}\text { Would you prefer if patient ask, } \\
\text { 1. Duration of hospital stay? }\end{array}$ & $\begin{array}{l}5.00 \\
0.54\end{array}$ & $\begin{array}{l}5.24 \\
0.74\end{array}$ & $\begin{array}{l}4.80 \\
0.85\end{array}$ & $\begin{array}{l}5.09 \\
0.74\end{array}$ & 12.01 & $0.00 * *$ \\
\hline 2. Duration of pain? & $\begin{array}{l}5.46 \\
0.56\end{array}$ & $\begin{array}{l}5.37 \\
0.61\end{array}$ & $\begin{array}{l}4.98 \\
0.80\end{array}$ & $\begin{array}{l}5.31 \\
0.66\end{array}$ & 14.05 & $0.00 * *$ \\
\hline $\begin{array}{l}\text { 3. Signs should look out for if } \\
\text { wound is not healing as it should? }\end{array}$ & $\begin{array}{l}5.22 \\
0.77\end{array}$ & $\begin{array}{l}4.58 \\
0.62\end{array}$ & $\begin{array}{l}4.60 \\
0.84\end{array}$ & $\begin{array}{l}4.74 \\
0.75\end{array}$ & 28.44 & $0.00 * *$ \\
\hline 4. Returning to normal activities? & $\begin{array}{l}5.29 \\
0.61\end{array}$ & $\begin{array}{l}4.94 \\
0.72\end{array}$ & $\begin{array}{l}5.03 \\
0.72\end{array}$ & $\begin{array}{l}5.04 \\
0.71\end{array}$ & 8.28 & $0.00 * *$ \\
\hline $\begin{array}{l}\text { 5. About the technique of } \\
\text { procedure done? }\end{array}$ & $\begin{array}{l}5.51 \\
0.74\end{array}$ & $\begin{array}{l}4.56 \\
3.41\end{array}$ & $\begin{array}{l}4.20 \\
0.96\end{array}$ & $\begin{array}{l}4.71 \\
2.63\end{array}$ & 6.31 & $0.00 * *$ \\
\hline Total & $\begin{array}{l}5.30 \\
0.28\end{array}$ & $\begin{array}{l}4.94 \\
0.71\end{array}$ & $\begin{array}{l}4.72 \\
0.49\end{array}$ & $\begin{array}{l}4.98 \\
0.62\end{array}$ & 7.52 & $0.00 * *$ \\
\hline
\end{tabular}

$* \mathrm{P}<0.05 \quad * * \mathrm{P}<0.01 \quad$ Source - survey data.

Table 2 displays descriptive statistics for selected HCPs attitudes towards factual questions as a HCP in CSHW and DSHW. Doctors, nurses and midwifes all were in agreement that they would support by responding to factual questions, although doctors seem to be strongly support compared with other two selected HCP categories. Midwives support least out of three selected HCPs in majority of questions still having a positive attitude. Only for one item nurses found significantly support more than doctors. ("Duration of hospital stay?", $\mathrm{P}<0.001$ ). 
Table 3 - Selected HCP's attitudes towards challenging questions in both CSHW and DSHW

\begin{tabular}{|c|c|c|c|c|c|c|}
\hline \multirow[t]{3}{*}{ Item Description } & Doctors & Nurses & Midwives & Total & $\mathbf{F}$ & $P$ value \\
\hline & $\begin{array}{l}n=94 \\
(24 \%)\end{array}$ & $\begin{array}{l}n=215 \\
(55 \%)\end{array}$ & $\begin{array}{c}n=82 \\
(21 \%)\end{array}$ & $\begin{array}{l}N=391 \\
(100 \%)\end{array}$ & & \\
\hline & $\begin{array}{c}\text { Mean } \\
\text { SD }\end{array}$ & $\begin{array}{l}\text { Mean } \\
\text { SD }\end{array}$ & $\begin{array}{l}\text { Mean } \\
\text { SD }\end{array}$ & $\begin{array}{c}\text { Mean } \\
\text { SD }\end{array}$ & & \\
\hline \multicolumn{7}{|l|}{$\begin{array}{l}\text { Challenging questions } \\
\text { (interactional behaviour) }\end{array}$} \\
\hline \multicolumn{7}{|l|}{ Would you prefer if patient ask, } \\
\hline $\begin{array}{l}\text { 6. Whether this is the correct } \\
\text { medication for me? }\end{array}$ & $\begin{array}{l}5.09 \\
0.71\end{array}$ & $\begin{array}{l}3.64 \\
0.78\end{array}$ & $\begin{array}{l}4.09 \\
0.81\end{array}$ & $\begin{array}{l}4.08 \\
0.97\end{array}$ & 114.63 & $0.00 * *$ \\
\hline 7. About your identification? & $\begin{array}{l}4.91 \\
0.69\end{array}$ & $\begin{array}{l}4.36 \\
0.74\end{array}$ & $\begin{array}{l}4.62 \\
0.95\end{array}$ & $\begin{array}{l}4.55 \\
0.81\end{array}$ & 16.34 & $0.00 * *$ \\
\hline 8. About your hand washing? & $\begin{array}{l}4.63 \\
1.00\end{array}$ & $\begin{array}{l}5.02 \\
0.67\end{array}$ & $\begin{array}{l}4.79 \\
0.85\end{array}$ & $\begin{array}{l}4.88 \\
0.81\end{array}$ & 8.35 & $0.00 * *$ \\
\hline $\begin{array}{l}\text { 9. About your experience on } \\
\text { particular task? }\end{array}$ & $\begin{array}{l}5.18 \\
0.73\end{array}$ & $\begin{array}{l}4.40 \\
0.57\end{array}$ & $\begin{array}{l}4.63 \\
0.85\end{array}$ & $\begin{array}{l}4.64 \\
0.75\end{array}$ & 41.75 & $0.00 * *$ \\
\hline $\begin{array}{l}\text { 10. Opinion on receiving } \\
\text { medication in her native language }\end{array}$ & $\begin{array}{l}5.22 \\
0.72\end{array}$ & $\begin{array}{l}4.72 \\
0.55\end{array}$ & $\begin{array}{l}4.84 \\
0.69\end{array}$ & $\begin{array}{l}4.86 \\
0.66\end{array}$ & 20.51 & $0.00 * *$ \\
\hline Total & $\begin{array}{l}5.01 \\
0.45\end{array}$ & $\begin{array}{l}4.43 \\
0.38\end{array}$ & $\begin{array}{l}4.59 \\
0.50\end{array}$ & $\begin{array}{l}4.60 \\
0.48\end{array}$ & 58.38 & $0.00 * *$ \\
\hline
\end{tabular}

Table 3 shows descriptive statistics for attitudes towards challenging questions as a HCP. All three selected HCPs were in general consensus that they would support by responding to challenging questions, although doctors seem to strongly support compared with other two selected HCP categories as a overall. Only for one item nurses found significantly support more than other two HCPs. (“About your hand washing?", $\mathrm{P}<0.001$ )

Table 4 - Selected HCP's attitudes towards notifying questions in both CSHW and DSHW

\begin{tabular}{|c|c|c|c|c|c|c|}
\hline \multirow[t]{3}{*}{ Item Description } & Doctors & Nurses & Midwives & Total & $\mathbf{F}$ & P value \\
\hline & $\begin{array}{l}\mathbf{n = 9 4} \\
(24 \%)\end{array}$ & $\begin{array}{l}\mathrm{n}=215 \\
(55 \%)\end{array}$ & $\mathrm{n}=82(21 \%)$ & $\begin{array}{l}\mathrm{N}=391 \\
(\mathbf{1 0 0 \%})\end{array}$ & & \\
\hline & Mean & $\begin{array}{l}\text { Mean } \\
\text { SD }\end{array}$ & $\begin{array}{l}\text { Mean } \\
\text { SD } \\
\end{array}$ & $\begin{array}{l}\text { Mean } \\
\text { SD } \\
\end{array}$ & & \\
\hline \multicolumn{7}{|l|}{$\begin{array}{l}\text { Notifying (interactional } \\
\text { behaviour) }\end{array}$} \\
\hline \multicolumn{7}{|l|}{ Would you like the patient notifying, } \\
\hline 11. About not receiving the results of a5. & 15.19 & 4.89 & 4.92 & 4.97 & & \\
\hline medical test? & 0.53 & 0.86 & 0.81 & 0.79 & 4.71 & $0.00 * *$ \\
\hline $\begin{array}{l}\text { 12. About an error that has occurred5 } \\
\text { during their care? }\end{array}$ & $\begin{array}{l}5.36 \\
0.63\end{array}$ & $\begin{array}{l}4.85 \\
0.64\end{array}$ & $\begin{array}{l}4.80 \\
0.72\end{array}$ & $\begin{array}{l}4.96 \\
0.69\end{array}$ & 2248 & $000 * *$ \\
\hline 13.If wound become infected & 5.26 & 4.81 & 4.98 & 4.95 & & \\
\hline
\end{tabular}




\begin{tabular}{|c|c|c|c|c|c|c|}
\hline & 0.69 & 0.70 & 0.98 & 0.78 & 11.34 & $0.00^{* *}$ \\
\hline $\begin{array}{l}\text { 14. About the correct site fo } \\
\text { surgery? }\end{array}$ & $\begin{array}{r}\text { he } 5.28 \\
0.63\end{array}$ & $\begin{array}{l}5.01 \\
0.93\end{array}$ & $\begin{array}{l}4.71 \\
1.05\end{array}$ & $\begin{array}{l}5.02 \\
0.91\end{array}$ & 8.73 & $0.00 * *$ \\
\hline Total & $\begin{array}{l}4.22 \\
0.32\end{array}$ & $\begin{array}{l}3.91 \\
0.34\end{array}$ & $\begin{array}{l}3.88 \\
0.48\end{array}$ & $\begin{array}{l}3.98 \\
0.39\end{array}$ & 25.14 & $0.00 * *$ \\
\hline
\end{tabular}

$* \mathrm{P}<0.05 \quad * * \mathrm{P}<0.01 \quad$ Source - survey data.

Table 4 shows descriptive statistics for items measuring selected HCPs attitudes towards above mentioned notifying questions as a HCP. Three categories of HCPs were in favour of supporting patients in notifying questions. According to post hoc analysis total means score difference between doctors-nurses and doctors-midwives were statistically significant $(\mathrm{P}<0.001)$. However total, mean score difference between nurses and midwives were not statistically significant as whole.

Table 5 - Selected HCP's attitudes towards information provision questions in both CSHW and DSHW

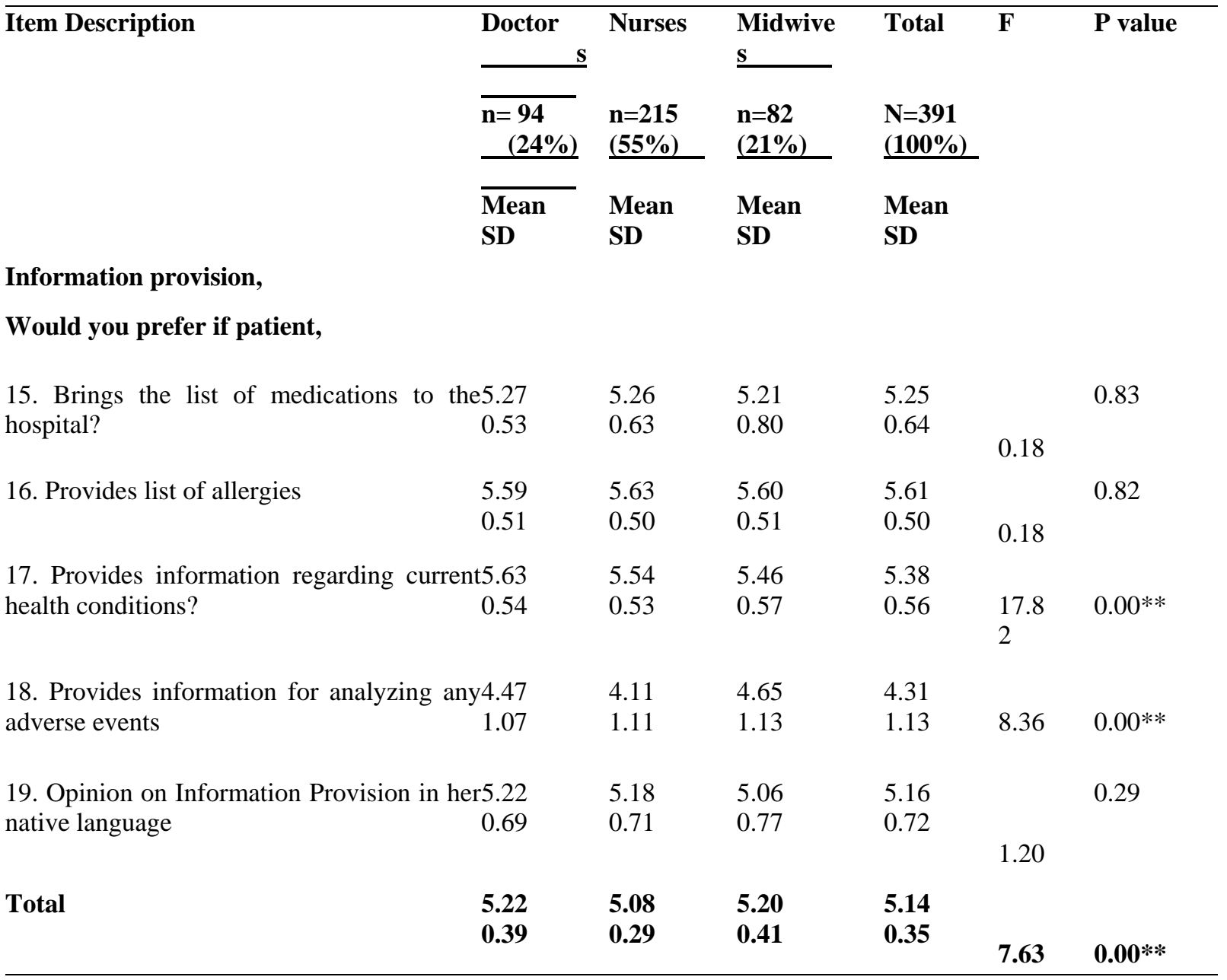

$* \mathrm{P}<0.05 \quad * * \mathrm{P}<0.01 \quad$ Source - survey data. 
Table 5 shows descriptive statistics for items measuring selected HCPs attitudes towards above mentioned information provision questions as a HCP. Difference in total means score between doctors and nurses were highly significant $(\mathrm{P}=<0.001)$ and difference between midwives and nurses were significant $(\mathrm{P}=$ $<0.05)$. In contrast doctors and midwives total means score difference were not significant.

Table 6 - Selected HCP's attitudes towards reporting incidents in both CSHW and DSHW

\begin{tabular}{|c|c|c|c|c|c|c|}
\hline \multirow[t]{2}{*}{ Item Description } & Doctors & Nurses & Midwives & Total & $\mathbf{F}$ & $P$ value \\
\hline & $\begin{array}{l}n=94 \\
(24 \%)\end{array}$ & $\begin{array}{l}n=215 \\
(55 \%)\end{array}$ & $n=82(21 \%)$ & $\begin{array}{l}N=391 \\
(100 \%)\end{array}$ & & \\
\hline
\end{tabular}

Incident reporting,

Would you prefer if the patient,

\begin{tabular}{|c|c|c|c|c|c|}
\hline $\begin{array}{lr}20 . \text { Reports to local authority/hospital3.97 } \\
\text { about adverse event? }\end{array}$ & $\begin{array}{l}4.26 \\
0.78\end{array}$ & $\begin{array}{l}4.21 \\
0.84\end{array}$ & $\begin{array}{l}4.18 \\
0.85\end{array}$ & 3.76 & $0.02 * *$ \\
\hline 21.Asks for a compensation? & $\begin{array}{l}2.11 \\
0.95\end{array}$ & $\begin{array}{l}2.57 \\
1.29\end{array}$ & $\begin{array}{l}2.27 \\
1.14\end{array}$ & 5.52 & $0.00 * *$ \\
\hline $\begin{array}{l}\text { 22.Divulges a medical error to public by } 1.69 \\
\text { mass media? }\end{array}$ & $\begin{array}{l}1.57 \\
0.88\end{array}$ & $\begin{array}{l}1.87 \\
1.08\end{array}$ & $\begin{array}{l}1.66 \\
0.94\end{array}$ & 3.10 & $0.04 *$ \\
\hline $\begin{array}{l}2.68 \\
0.90\end{array}$ & $\begin{array}{l}2.65 \\
0.63\end{array}$ & $\begin{array}{l}2.89 \\
0.87\end{array}$ & $\begin{array}{l}2.70 \\
0.76\end{array}$ & 2.99 & $0.05 *$ \\
\hline
\end{tabular}

$*$ P $\quad * * 05 \quad$ Source - survey data.

Table 6 Illustrates descriptive statistics for items measuring selected HCPs attitudes towards above mentioned incident reporting questions as a $\mathrm{HCP}$. In contrast to previous mentioned components, this clearly shows negative attitudes for incident reporting component among three groups of HCPs Total means score less than 3.0 across three categories. (Likert scale ranging from 1-6).

\section{DISCUSSION}

To the best of our knowledge, this could be the first research done in Sri Lanka on health care professional's perspectives on patient involvement in patient safety as a Health Care Professional (HCP) . Our research finding suggested that HCPs' attitudes can be varied with type of behavior (interactional and noninteractional behavior), type of HCP category and participants own professional role (as a HCP).. There were some differences in HCPs' perspectives in two studied health care institutions.

Sociodemographic data

Sociodemographic results (Table 01) showed that there were $94(24 \%)$ doctors, $215(55 \%)$ nurses and $82(21 \%)$ midwives with the total of 391. Current working experience showed that majority of three selected HCPs' were in less than 05 year category. Professional qualification showed that all midwives holding a diploma (100\%), nurses were holding a basic degree $(100 \%)$ and majority of doctors holding an undergraduate degree $(95 \%)$. There was no significant difference noted among participant characteristics between two health care institutions.

Perspectives on patient involvement in patient safety as a HCP

First step (survey 01) was to HCPs' perspectives on patient involvement in patient safety as a HCP. Survey-01 was consisting of 05 components they were subdivided in to 22 items. Five components were,

1. Factual questions -05 items (interactional behavior)

2. Challenging questions-04 items (interactional behavior)

3. Notifying questions-05 items (interactional behavior)

4. Information questions -05 items (noninteractional behavior) 


\section{Reporting questions - 03 items (noninteractional behavior)}

Overall, three categories of selected health care professionals showed positive attitudes towards patient involvement in patient safety as a $\mathrm{HCP}$ for four components (Table $2-5$ ). Considering three categories of HCP, doctors found to have more willingness in supporting compared with other two HCP categories. Midwives were the least supporting group still with positive attitude. Remarkably, incident reporting component (Table 6) showed negative attitudes across three professional categories (mean ranges from 2.65-2.89, $\mathrm{P}<0.001$ ).

It is interesting to compare the our research findings with the research done by Rachel E Davis et al.[6],[7],[8],[9]Their research findings showed that two HCPs' groups (doctors and nurses) had positive willingness towards patient involvement in patient safety comparable to our result. Incident reporting component in their research showed positive attitudes, in contrast to our research findings. They found that nurses showed more willingness compared with doctors in contrary to our findings.

Analysis of five components as a HCP in our study showed that doctors were scoring more compare to other two groups in general. Nurses got exceptionally higher score only in one item of factual statements that was "duration of hospitals stay" which was statistically significant also (Table 2). Second component was the challenging questions (interactional behavior) where doctors scored highest as previous component. However, nurses scored least in this component except one that was

"about your hand washing"? (Table 3). This behavior could be due to nurses regular involvement in such activity. Rachel $\mathrm{E}$ Davis et al, found similar results with nurses showing exceptionally positive attitudes for hand washing compared with doctors. Another study which was conducted in U K showed that nursing officers assume that it is very important to hand washing before caring a patient.[10] Perhaps this could be due to nursing officers are more willing to help patients in asking such type of question. Next component, notifying questions (Table 4 ) showed overall results similar to factual questions results. Information provision statements also displayed positive attitudes of all three HCP categories (Table 5 ). Doctors and nurses were scored highest and lowest respectively.

Study carried out at Nepal found that healthcare professionals have positive attitudes towards adverse drug reactions reporting in contrast to our results even though from same region. Increasing awareness among healthcare professionals, training and collaboration would likely to improve reporting.[11]

Health care professionals might give opportunities to the patient participation in different degrees depending on clinical situation. Doctors were more willing to allow patient participation when dealing with psychological rather than physical issues. In contrast; patients were less participating when a therapeutic or a diagnostic procedure is performed.[12] Health care personal attitudes can play important role in deciding on value of the patient's opinion. Research conducted in involving 600 obstetricians on their opinion about elective, client requested cesarean operation found that many obstetricians believed women's had a right to request for caesarian section, in contrast $25 \%$ who believed that women didn't have a such a right.[13]

\section{Limitations of the study}

There were few limitations in our research. First, our sample was confined to two women's hospitals in Colombo and only involving obstetric and gynaecological specialities. Therefore, our research findings need to be generalized with caution as one speciality of medicine was involved. Therefore, much larger sample with different medical specialities needed before applying for general population.

\section{CONCLUSIONS AND RECOMMENDATIONS}

Conclusions

Our research findings suggest that three categories of selected HCPs' were willing to support patient involvement in patient safety as a HCP . Regarding incident reporting all three selected HCPs' were had negative attitude as a HCP.

\section{Recommendations}

1. Negative attitudes on incident reporting need to consider as a priority. It's necessary to emphasize HCPs' about the nature of incident reporting that is as a fact finding process rather than fault finding procedure. Establishing a no blame culture in health care institutions will play key role in changing their behaviour. Updating or developing guidelines on incident reporting and distributing among HCPs'can encourage their participation in incident reporting.

2. Periodically (e.g. quarterly, annually) conducting training and educational program on patient safety allow HCPs' staff to refresh and update current knowledge on patient safety. Induction programs should have a patient safety component which allow new comers to familiar with patient safety issues at the beginning.

3. Establishing active on going patient feedback system allow HCPs' to response to their failures and mistakes timely and efficiently. This will indirectly create patient's confidence about their health care providers.

4. As this research was confined to obstetrics and gynaecological field it is mandatory to conduct research involving other medical fields with much larger samples. This will increase the generalizability and applicability in future. Other component is that it is necessary to conduct studies involving actual patients to get the real perspectives on patient safety from their eye.

\section{REFERENCES}

[1] World Alliance for Patient Safety forwarered programme (2005) Available at: www.who.int/patientsafety/en/brochure_final.pdf (Accessed: 18 May 2017).

[2] National Library of Medicine (2002) National Institute of Health, History of Medicine: Greek Medicine

[3] Vincent, C. (2011) Patient Safety, 2nd edn. London: Willy -Blackwell.

[4] Brennan, T.A., Leape, L.L. and Laird, N (1991) 'Incidence of adverse events and negligence in hospitalized patients: results of the Harvard Medical Practice Study'. New England Journal of Medicine, 324 (6), pp. 370-377.

[5] Jha, A.K., Larizgoitia I. and Audera-Lopez. C, (2013) 'The global burden of unsafe medical care an observational study', British Medical Journal, vol 22, no10, pp 809- 815 . 
[6] Davis R, (2007) 'Patient involvement in patient safety: what factors influence patient participation and engagement', American journal of obstetrics and gynecology, vo10, no3, pp. 259-267

[7] Davis, R., Koutantji, M. and Vincent, C. (2008) 'How willing are patients to question healthcare staff on issues related to the quality and safety of their healthcare? An exploratory study', QSHC, 17, pp. 90-96.

[8] Davis, R., Sevdalis, N. and Jacklin, R. (2007) 'Patient involvement in safety: What factors influence patient participation and engagement. Health Expectations'

[9] Davis, R., Sevdalis, N. and Vincent, C. (2011) 'Patient involvement in safety: how willing are patients to participate?', QSHC, 20, pp. 108-114.

[10] Stein, A.D., Makarawo, T, P. and Ahmad, M.F. (2003) 'A survey of doctors' and nurses' knowledge, attitudes and compliance with infection control guidelines in Birmingham teaching hospitals', J Hosp Infect, 54, pp. 68-73.

[11] Santosh, K.C., Tragulpiankit, P., Gorsanan, S. and Edwards, I.R. (2013) 'Attitudes among healthcare professionals to the reporting of adverse drug reactions in Nepal', BMC Pharmacology and Toxicology, pp. 14-16.
[12] Van den Brink-Muinen, A., van Dulmen, S.M., de Haes, H.C., Visser, A.P., Schellevis, F.G. and Bensing, J.M. (2006) 'Has patients' involvement in the decision- making process changed over time?', Health Expect, 9(4), pp. 333342.

[13] Bettes, B.A., Coleman V.H. and Zinberg, S. (2007) 'Cesarean delivery on maternal request: obstetrician-gynecologists' knowledge, perception, and practice patterns', Obstret Gynaecol, 109(1), pp. 57-66.

\section{AUTHORS}

First Author - Dr W D K Wijesinghe, Trainee, MD in Medical Administration, Post Graduate Institute of Medicine. University of Colombo.

Second Author - Dr Sathasivam Sridharan, Deputy Director General - Planning, Ministry of Health, Colombo, Sri Lanka 